\title{
Gwenola Bargain, Normativité économique et droit du
} travail

Préface d'Alain Supiot, Issy-les-Moulineaux, LGDJ-Lextenso, coll. « Droit \& économie ", 2014

\section{Thierry Kirat}

\section{(2) OpenEdition}

\section{Journals}

Édition électronique

URL : http://journals.openedition.org/travailemploi/7200

DOI : $10.4000 /$ travailemploi.7200

ISSN : 1775-416X

Éditeur

DARES - Ministère du Travail

Édition imprimée

Date de publication : 1 juillet 2016

Pagination : 133-137

ISSN : 0224-4365

\section{Référence électronique}

Thierry Kirat, «Gwenola Bargain, Normativité économique et droit du travail », Travail et Emploi [En ligne], 147 | juillet-septembre 2016, mis en ligne le 11 juillet 2019, consulté le 24 septembre 2020. URL http://journals.openedition.org/travailemploi/7200 ; DOI : https://doi.org/10.4000/travailemploi.7200 


\section{Normativité économique et droit du travail}

\section{Gwenola Bargain}

Préface d'Alain Supiot, Issy-les-Moulineaux, LGDJ-Lextenso, coll. « Droit \& économie », 2014, $535 \mathrm{p}$.

\section{Thierry Kirat"}

Voilà un ouvrage, couronné par un prix décerné par l'Association française de droit du travail et de la sécurité sociale, qui ne manque ni d'ampleur ni de profondeur. Issu de la thèse de doctorat en droit que son auteure, agrégée d'économie-gestion, a réalisé sous la direction d'Alain Supiot, il s'intéresse à la manière dont l'analyse économique colonise le droit du travail. Bel exemple de la combinaison heureuse de compétences dans deux disciplines - le droit et l'économie -, il témoigne de la valeur ajoutée de la pluridisciplinarité, à savoir la capacité à produire des connaissances que les disciplines prises séparément seraient dans l'incapacité de réaliser.

De quoi s'agit-il dans cet ouvrage ? De repérer, avec une grande minutie, les « ressorts de la normativité économique en droit du travail », ce qui est l'objet de la deuxième partie de l'ouvrage. Pour procéder à ce repérage, à la fois en droit européen et en droit interne, l'auteure nous invite à une enquête préalable sur les « fondements scientifiques en droit du travail », c'est-à-dire à faire connaissance, pour les lecteurs qui n'ont pas de « background » en économie, avec les représentations que les modèles théoriques se font du travail et de ses règles. De fait, les discours économiques sur le droit du travail, la protection de l'emploi, les institutions du marché du travail, s'érigent en discours normatifs concurrents de la normativité juridique classique. Plus encore, ils trouvent certains chemins pour s'y immiscer et contribuent à la transformer.

Le premier titre de la première partie s'attache à ce que l'auteure appelle l' « ordre contractuel », qui renvoie plus précisément à l'analyse économique des contrats lorsqu'elle est appliquée à la relation de travail. L'auteure y montre que l'analyse économique « construit le contrat » selon ses propres termes et que cette construction devient le modèle à l'aune duquel les règles de droit sont évaluées. Différentes notions sont abordées dans les deux chapitres de ce titre 1 : les préférences données, l'opportunisme, les incitations, la rupture efficiente du contrat, l'incomplétude contractuelle et les règles supplétives. Du point de vue de l'analyse économique, Gwenola Bargain fait preuve de beaucoup de maîtrise. Ses développements sont d'autant plus originaux

\footnotetext{
* Institut de recherche interdisciplinaire en sciences sociales (Irisso), université Paris Dauphine.
} 
qu'ils donnent à voir la méthodologie - implicite car rarement formalisée - de la pensée économique appliquée au droit : construire un modèle théorique, faire émerger les failles de la coordination des comportements, puis poser que les solutions juridiques (comme l'intervention du juge) pourraient être remplacées par des solutions économiques (comme des mécanismes incitatifs). Le deuxième titre de la première partie se déplace sur le terrain de la concurrence ( «L'ordre concurrentiel »). La dimension juridique y est plus affirmée que dans le premier titre. L'auteure met surtout l'accent sur l'intégration par le marché dans l'Union européenne (UE), et sur les relations entre le droit économique du marché européen et le droit du travail. Elle reconnaît que « [le concept d'] ordre concurrentiel ne relève pas, directement, de l'analyse économique, à tout le moins néoclassique, et qu'il n'est pas non plus utilisé au sein de l'analyse économique du droit » (p. 118). En utilisant ce concept dont la paternité revient aux juristes de droit économique de l'École de Nice (les regrettés Antoine Pirovano et Laurence Boy), elle en fait le socle d'une analyse, là encore très précise, de la nouvelle donne que l'institution du marché européen, couplée à la construction de libertés économiques fondamentales, représente pour les réglementations nationales du travail et l'exercice des droits sociaux. En substance, les libertés économiques dans l'UE l'emportent sur les droits sociaux conçus initialement comme autonomes par rapport au marché. L'auteure suit à la trace la jurisprudence de la Cour de justice de l'Union européenne à propos des relations qu'elle établit entre, d'une part, les règles de la concurrence et les « libertés économiques » (les libertés d'établissement et de prestation de service) et, d'autre part, le droit du travail, qu'il s'agisse de conventions collectives ou d'actions collectives. G. Bargain insiste longuement sur les arrêts Viking et Laval rendus par la Cour de justice des communautés européennes (CJCE), ainsi que sur les conclusions des avocats généraux. Alors qu'en 1999, dans l'arrêt Albany, la Cour décide qu'un accord collectif échappe aux règles de concurrence, dans les arrêts Viking et Laval rendus en 2007 portant sur des actions collectives (des grèves) et leur conformité à la liberté d'établissement et de prestation de service ${ }^{1}$, elle décide que les nécessités marchandes (le marché intérieur) l'emportent sur l'exercice des droits sociaux. Outre l'analyse attentive de la jurisprudence européenne, l'auteure recherche l'influence de l'analyse économique dans la remise en cause de l'autonomie du champ social et du champ économique contenue dans l'arrêt Albany : en substance, l'analyse économique tend à évaluer les effets des conventions collectives sur le bien-être (p. 133). Maintenir l'autonomie de la négociation collective a pour conséquence, inacceptable pour les

\footnotetext{
1. Le fond de l'arrêt Viking (CJCE, 11 décembre 2007) porte sur la contestation par un syndicat finlandais de travailleurs du secteur des transports de la décision d'une compagnie maritime de passer d'un pavillon finlandais à un pavillon estonien et de bénéficier ainsi d'une convention collective plus avantageuse, en termes de coûts salariaux, en Estonie. Le syndicat finlandais a menacé de faire grève et le syndicat international des travailleurs du transport a recommandé à ses affiliés, dans d'autres pays, de négocier avec la société Viking. Dans l'arrêt Laval (CJCE, 18 décembre 2007), le fond de l'affaire concerne des travailleurs détachés : une société de droit letton ayant obtenu un contrat de construction en Suède, elle détache des travailleurs lettons dans sa filiale suédoise et refuse de respecter la législation suédoise sur le salaire minimum et les conditions de travail ; elle refuse également de signer une convention collective. Les syndicats suédois lancent une grève pour que les travailleurs lettons bénéficient du droit suédois.
} 
économistes, de faire échapper ce dispositif juridique à l'évaluation économique (p. 134). Est-il utile de préciser que l'analyse économique dont il est question relève de l'économie standard?

Venons-en à la deuxième partie de l'ouvrage, qui porte sur « les ressorts de la normativité économique en droit du travail ». Son intention est de faire apparaître la force qui «fait agir » cette normativité au sein de la matière juridique (p. 243). L'auteure place plus précisément la focale sur deux questions successives : d'abord le juge et son raisonnement dans le titre 1, puis l'évolution des sources du droit du travail dans le titre 2 .

Susceptible d'être ouvert à la normativité économique, le raisonnement du juge peut l'être de deux manières, qui sont soigneusement distinguées par l'auteure. L' " approche formelle du jugement d'économie », exposée dans le chapitre 1, renvoie aux situations dans lesquelles « le raisonnement du juge emprunte au raisonnement économique » (p. 246) : par le recours à l'analyse coûts-avantages, ou par l'adoption d'un modèle économique d'évaluation des droits. L' " approche matérielle », présentée dans le chapitre 2, signifie que les intérêts matérialisés, des salariés et des entreprises, sont pris en considération. Précisant que ces approches ne sont pas exclusives, G. Bargain les travaille en profondeur et en présente de nombreuses illustrations en droit positif interne et en droit européen, comme nous allons le voir.

Dans la perspective formelle, ce qui est en jeu est la « recherche d'économies », c'est-à-dire l'optimisation économique des droits via le recours à l'analyse coûtsavantages. Non seulement l'auteure trouve des exemples de cette recherche d'économies dans les systèmes juridiques américain et anglais, mais elle en révèle des traces en droit du travail français, plus précisément dans les dispositions légales relatives aux conditions de travail des travailleurs handicapés qui mettent en balance les avantages attendus de l'aménagement du poste de travail et les coûts de telles mesures pour les employeurs. Dans le système juridique français, c'est dans l'utilisation judiciaire de la «théorie du bilan » issue de la jurisprudence administrative ${ }^{2}$ que l'auteure poursuit sa recherche de «marqueurs » de jugements d'économie : elle en découvre ainsi dans le contrôle judiciaire du motif économique du licenciement, c'est-à-dire dans la mise en balance des coûts subis par le salarié licencié « au regard de l'intérêt procuré à l'entreprise par la mesure en cause » (p. 267), dans la limite imposée par la Cour de cassation de ne pas formuler de jugement sur l'opportunité des décisions des employeurs. Le standard du « raisonnable » en est également porteur : emprunté par le droit de l'UE aux systèmes juridiques anglo-américains, ce standard au contenu variable se transmue en référence à un modèle de calcul rationnel, qui soupèse les avantages et les coûts des solutions juridiques. Alors qu'à l'origine, il servait au juge à apprécier ce qui peut être

2. Forgée par le Conseil d'État dans son arrêt «Ville nouvelle Est » en 1971, elle peut être considérée comme une forme d'analyse coûts-avantages. Elle consiste à estimer, pour une opération d'aménagement d'utilité publique, « si les atteintes à la propriété privée, le coût financier et éventuellement les inconvénients d'ordre social qu'elle comporte ne sont pas excessifs eu égard à l'intérêt qu'elle présente » (pour la collectivité). 
valablement attendu d'une personne « raisonnable », il a accueilli progressivement des éléments d'appréciation fondés sur le calcul coûts-avantages.

Au terme de son analyse de la jurisprudence française, l'auteure conclut qu'en définitive, dans la perspective juridique, l'analyse coûts-avantages « vise à rendre compte des multiples intérêts en présence » (p. 272) ; le vecteur de cette pluralité des intérêts est le contrôle de proportionnalité. Or, ce dernier est compatible avec une logique de conciliation des intérêts, qui s'exerce au détriment de la teneur des droits qui se confrontent. En témoigne une ligne jurisprudentielle qui retient une véritable approche marchande des droits, qui s'accompagne de la renonciation à une liberté fondamentale.

L'approche matérielle du jugement d'économie déporte l'analyse sur le terrain de la représentation du travail : dès lors que les enjeux juridiques sont placés sous l'égide des nécessités et des besoins du marché, le travail devient marchandise. Les activités juridiques sont en effet parfois déterminées par une représentation marchande du travail, qui devient ainsi source de normativité. L'évaluation marchande passe par plusieurs canaux : d'abord le juge apprécie l'utilité d'une mesure au regard de l'état du marché, par exemple lorsqu'il doit statuer sur une clause de non-concurrence ou sur un plan de sauvegarde de la compétitivité de l'entreprise. Dans ces cas, il s'agit de préserver la situation concurrentielle de l'entreprise. Ensuite, le juge surpondère le marché du travail par rapport à l'activité de travail stricto sensu. Ainsi, en validant une différence de traitement entre salariés locaux et salariés étrangers dans l'arrêt Synchrotron, la Chambre sociale de la Cour de cassation soutenait en 2005 que les salaires plus élevés des travailleurs étrangers avaient aussi pour but de «faciliter l'embauche de salariés ressortissants non français des parties contractantes afin de contribuer à la création d'un pôle d'excellence scientifique international » (p. 335, italiques originales). La rémunération n'est plus tant la rétribution du travail qu'un mécanisme incitatif. Malgré tout, G. Bargain conclut que, in fine, « la considération de l'état du marché du travail n'est pas omniprésente dans l'appréciation du juge » (p. 342).

Le dernier titre de l'ouvrage est consacré aux relations entre la normativité économique et l'évolution des sources sur lesquelles s'appuie le droit du travail. Un premier chapitre analyse avec précision « le désordre du droit du travail »; le suivant, « la recomposition de la distinction public-privé ». Le chapitre 1 met en évidence les tendances lourdes de la négociation collective et du rapport entre le droit et le contrat. C'est dans le mouvement de décentralisation de la négociation sociale au niveau de l'entreprise que l'influence des préconisations émise par des économistes depuis quinze ans est la plus nettement marquée : la gestion pragmatique du temps de travail par les accords d'entreprise correspond à l'objectif d'élaborer de manière décentralisée des règles adaptées à la situation économique. La règle négociée prend le pas sur le droit réglementaire. Le paysage juridique qui se dessine est celui d'un système où le supplétif devient le principe organisateur : la loi (ou la convention de branche) ne s'applique qu'à défaut d'accord d'entreprise. Le principe de faveur - qui permet aux 
salariés de bénéficier de la norme la plus favorable parmi celles qui s'appliquent - et l'ordre public social sont mis à mal. La protection des droits est désormais repoussée aux confins du droit négocié. Dans le chapitre sur la recomposition de la distinction public-privé, G. Bargain met en avant les marges d'autonomie reconnues aux acteurs privés tout en réaffirmant les droits sociaux fondamentaux et la constitutionnalisation du droit du travail. En résumé, alors que la libéralisation des règlements économiques au sein de l'UE est de nature à fragiliser « les mécanismes juridiques de solidarité des travailleurs à l'échelle de l'Union européenne », les règles du droit du travail, même négociées, auraient quant à elles par essence « une capacité d'entrave du marché » (p. 441). Dans ce contexte, l'affirmation des droits sociaux fondamentaux peut servir de base à la reconstruction, dont le principe est défendu par l'auteure, d'une représentation de l'action collective qui imposerait « un horizon normatif plus soucieux de la capacité d'exercice des libertés des individus » (p. 466), entendue en référence à la théorie des capacités d'Amartya Sen.

Somme impressionnante, de très grande qualité, consacrée au recensement et à l'analyse de l'influence des catégories normatives des modèles économiques, l'ouvrage de G. Bargain montre bien que ce qui est en jeu n'est pas rien : l'emprise d'une normativité marchande qui promeut une représentation du marché dans laquelle le travail n'est plus qu' une marchandise, qui réduit les comportements à des questions d'incitation, et qui prône une reconfiguration des relations professionnelles au nom du libre jeu du marché. L'ouvrage est déjà conséquent, et on peine à déceler des failles ou des limites. Peut-être peut-on simplement regretter que la proposition de reconstruire une analyse économique du droit du travail sur la base de la théorie des capacités d'A. Sen n'ait pas été poussée plus loin par l'auteure. Mais cela rend le lecteur d'autant plus impatient de lire les futurs travaux de G. Bargain. 